\title{
Immobilization of glucoamylase on stimuli-sensitive macroporous semi-interpenetrating stimuli-sensitive hydrogel carriers*
}

\author{
Li-Xia Li ${ }^{\dagger}$, Yi Yang, Fang Zhu and Xiang-Yang Wu \\ School of the Environment and Safety Engineering, \\ Jiangsu University \\ Zhenjiang, 212013, China \\ ${ }^{\dagger}$ E-mail: qingpipa@163.com
}

\begin{abstract}
Based on the special phase transition property of copolymer hydrogel of $\mathrm{N}$-is opropylacrylamide, the immobilization of glucoamylase on five macro porous semi-interpenetrating stimuli-sensitive hydrogel carriers was studied. Four good preparations were screened, i.e. GelNIPAm/PAEMA-2.5, Gel NAEP/PAEMA-2.5, Gel NAP/PAEMA-2.5 and Gel NDEP/PAEMA-2.5, the immobilization yield ${ }^{\circ}$ Cactivity) of them were $11.4 \%, 12.7 \%, 14.9 \%$ and $15.3 \%$ respectively under optimal conditions. The most preferred temperature of these immobilized glucoamylase was $30{ }^{\circ} \mathrm{C}$, and the adapted $\mathrm{pH}$ was about $\mathrm{pH}$ 4.5. The activities of the latter three photo-sensitive preparations could be adjusted by UV irradiation during their phase transition. After month storage, the activity retention rate was no less than $80 \%$ for every one preparations cross-linked with 2.5 vol \% of glutaraldehyde.

Keywords: Immobilization; Glucoamylase; Stimuli-sensitive hydrogel carriers; Thermo-/light-sensitive.
\end{abstract}

\section{Introduction}

Enzyme catalytic reaction is atomic reaction under mild conditions in water with high rates, least consumption of energy, and with no side reaction so that it is a focus of scholar's concerning. With the development of knowledge and technique, enzymes are involved in a more wide variety of applications such as food industries, textiles industries, chemical manufacture and other important industries [1]. However, there are some drawback for the use of enzyme such as the problem of purification and continuous operation, the lack of long-term stability and the difficulty in their recovery and reuse. Enzyme immobilization has been developed subsequently to overcome these insufficiencies [2]. In the case of carriers, a lot of various materials including inorganic materials, natural

\footnotetext{
* This work is supported by Collaborative Innovation Center of Technology and Material of Water Treatment, Jiangsu Postdoctoral Science Foundation (1402072B).
} 
polymers and synthetic polymers were reported [3]. $\mathrm{N}$-is opropylacrylamide ${ }^{\circ} \mathrm{CNIP} \mathrm{Am}$ ) copolymer is a kind of synthetic polymer support which was studied recently, which has a lower critical solution temperature ${ }^{\circ}$ CLCST) [4]. Below the LCST, the polymer is swollen and hydrated, whereas above the LCST, the polymer is shrunk which render it a collapsed and dehydrated state. The phase transition is reversible. When using the copolymer as carrier, it is possible to reversibly control the reaction by varying the temperature, gaining catalysis on demand. So, stimuli-responsive copolymer of NIPAm aroused many interests in many science research fields [5]. In the case of enzyme immobilization, immobilization of glucoamylase, trypsin [6], lipase [7], glucose oxidase [8] and pepsin [9] on homogeneous poly ${ }^{\circ} \mathrm{CNIPAm}$ ) or NIPAm copolymers were reported in recent years.

In our previous research work, several good stimuli-sensitive hydrogel were synthesized and screened successfully [10]. Based on this, one thermosensitive, GelNIPAm/PAEMA, and three kinds of themo-/light-sensitive macroporous semi-interpenetrating hydrogel carriers, GelNAEP/PAEMA, GelNAP/PAEMA and GelNDEP/PAEMA, were prepared. Then glucoamylases were immobilized on these carriers referring to document [11]. Glucoamylases were first adsorbed to the inside of the carriers during the phase transition from shrink to swelling state, and then were fixed by cross linking reaction using glutaraldehyde as cross-linking agent. Next, bound protein, specific activity of protein, immobilization yield ${ }^{\circ}$ Cactivity), and storage stability of these immobilized glucoamylases were studied carefully. The affection of temperature, UV light and $\mathrm{pH}$ on the activity and storage ability were also studied.

\section{Materials and Methods}

\subsection{Materials}

Liquid glucoamylase ${ }^{\circ} \mathrm{CCAS}$ : 9032-08-0) was purchased from Aladdin Industrial Corporation ${ }^{\circ} \mathrm{CShang} \mathrm{Hai}$ ). $\mathrm{N}$-isopropylacrylamide ${ }^{\circ} \mathrm{CNIPAm}, \mathrm{J} \& \mathrm{~K}$ Chemical Ltd., Shanghai, China) was used after purifying by recrystallization in mixed solution of hexane and toluene ${ }^{\circ} \mathrm{C} 40 / 60$ vol \%) and dried under a vacuum. BCA Protein Assay Kit and GOD glucose assay kit were purchased from Shanghai Chao Yan Biotechnology Corporation. Other materials were all commercial available and were used without further purification.

\subsection{Preparation of carriers}

The mixture of HEMA ${ }^{\circ} \mathrm{C} 1 \mathrm{~g}$ ), DMSO ${ }^{\circ} \mathrm{C} 4.5 \mathrm{~g}$ ) and $1 \mathrm{wt} \%$ of AIBN was heated to $60^{\circ} \mathrm{C}$ for $6 \mathrm{~h}$ under stirring. Line poly HEMA was extracted with aether from the product mixture and then was dried under vacuum. Next, a mixture of 
PHEMA and ethylenediamine was heated to reflux for 4 hour. Then, the redundant ethylenediamine was evaporated together with methane using rotary evaporator. The ploy ${ }^{\circ} \mathrm{C} 2$-amidoethyl methacrylamide) ${ }^{\circ} \mathrm{CPAEMA}$ ) was obtained.

PEG, NIPAm, co monomer, PAEMA or PHEMA, MBAm and AIBN were dissolved in DMSO successively. The mixture was degassed by bubbling with nitrogen for $15 \mathrm{~min}$ and then transferred into glass capillaries with $1 \mathrm{~mm}$ inner diameter and were heated to $60{ }^{\circ} \mathrm{C}$ for $6 \mathrm{~h}$. The gels were taken out and immersed in a lot of deionized water for at least two weeks in order to wash away DMSO, PEG and unreacted monomers and so on. The water was renewed daily. The feed compositions of copolymer hydrogels were showed in Table 1.

Table 1 Feed composition of copolymer hydrogels

\begin{tabular}{llllllll}
\hline Hydrogels & EGg & $\begin{array}{l}\text { AEMA/ } \\
\text { HEMA,g }\end{array}$ & IPAmg & 'omonomer ${ }^{\mathrm{a}} \mathrm{g}$ & IBAmg & IBN & iMSOml \\
\hline $\begin{array}{l}\text { GelNIPAm- } \\
\text { PHEMA }\end{array}$ & 0.15 & 0.075 & 0.151 & 0 & 0.004 & 0.005 & 0.6 \\
$\begin{array}{l}\text { GelNIPAm- } \\
\text { PAEMA }\end{array}$ & 0.15 & 0.075 & 0.151 & 0 & 0.004 & 0.005 & 0.6 \\
$\begin{array}{l}\text { GelNDEP- } \\
\text { PAEMA }\end{array}$ & 0.15 & 0.075 & 0.151 & $\begin{array}{l}0.0068 \\
\text { DMAE-EAPP }\end{array}$ & 0.004 & 0.005 & 0.6 \\
$\begin{array}{l}\text { GelNAEP- } \\
\text { PAEMA }\end{array}$ & 0.15 & 0.075 & 0.151 & $\begin{array}{l}0.005 \\
\text { AEPABA }\end{array}$ & 0.004 & 0.005 & 0.6 \\
$\begin{array}{l}\text { GelNAP- } \\
\text { PAEMA }\end{array}$ & 0.15 & 0.075 & 0.151 & $\begin{array}{l}0.0044 \\
\text { APABA }\end{array}$ & 0.004 & 0.005 & 0.6 \\
\hline
\end{tabular}

${ }^{a}$ Comonomers, DMAE-EAPP, APEABA and APABA, were prepared according our report [5].

\subsection{Immobilization}

First, swelling hydrogel carrier was placed in deionized water at $45^{\circ} \mathrm{C}$ so that it was shrunken, and then was translated into the solution of glucoamylase in $45^{\circ} \mathrm{C}$ $0.1 \mathrm{M}$ phosphate buffer ${ }^{\circ} \mathrm{C} \mathrm{pH} 7.0$ ), 50mg/ml, and stayed for $20 \mathrm{~min}$ with slightly stirring. Then the mixture was cooled down to $4^{\circ} \mathrm{C}$ quickly and stayed for $1 \mathrm{~h}$. Next, the mixture was heated to $45^{\circ} \mathrm{C}$ again so that the hydrogel carrier was dehydrated. The free enzyme solution was sucked out with a glass sinter and was analyzed for the presence of protein. The amount of bound protein was calculated as a result of subtraction of the amount used for immobilization. And the shrunk carrier was divided into three parts.

Next, solutions of glutaraldehyde ${ }^{\circ} \mathrm{C} 0 ; 1 ; 2.5$ vol \%) in $0.1 \quad \mathrm{M}$ phosphate buffer ${ }^{\circ} \mathrm{C} \mathrm{pH} 7.0$ ) were prepared and added to the above carriers at $20^{\circ} \mathrm{C}$. After $15 \mathrm{~min}$ of reaction, carriers were took out and washed with $0.1 \mathrm{M}$ 
phosphate buffer ${ }^{\circ} \mathrm{CpH}$ 7.0) for three times. Last, the preparations were immersed in $0.05 \mathrm{M}$ tris- $\mathrm{HCl}$ buffer ${ }^{\circ} \mathrm{CpH} 8.0$ ) and stored for $24 \mathrm{~h}$ at $4{ }^{\circ} \mathrm{C}$.

\subsection{Activity of free glucoamylase and immobilized preparations}

First, $2.5 \mathrm{wt}$. \% soluble starch was gelatinized in water of $100{ }^{\circ} \mathrm{C}$ for $15 \mathrm{~min}$.

Free glucoamylase activity was assayed by GOD assay kit. $1 \mathrm{ml}$ solution of glucoamylase was added into $1 \mathrm{ml}$ of gelatinized starch solution. After $5 \mathrm{~min}$ of incubation at some temperature, $20 \mu \mathrm{l}$ mixtures was taken and added to $780 \mu \mathrm{l}$ of working solution at $30^{\circ} \mathrm{C}$. After $25 \mathrm{~min}$ of reaction, the mixture was diluted by distilled water to a certain extent when the glucose content was less than $20 \mathrm{mmol} / \mathrm{L}$. The absorbance ${ }^{\circ} \mathrm{C} 553 \mathrm{~nm}$ ) was measured. The glucose content was calculated from the absorbance on the base of glucose standard absorbance and dilution ratio. The enzyme activity unit ${ }^{\circ} \mathrm{CU}$ ) was defined as the amount of enzyme liberating $1 \mu \mathrm{mol}$ glucose per minute under assay conditions.

Immobilized preparations ${ }^{\circ} \mathrm{C} 1 \mathrm{~mL}$ ), swelling sufficiently in $0.05 \mathrm{M}$ acetate buffer ${ }^{\circ} \mathrm{CpH} 4.5$ ) was heated to a scheduled temperature. Gelatinized starch solution ${ }^{\circ} \mathrm{C} 1 \mathrm{~mL}$ ) was added into the preparations and $20 \mu 1$ mixtures were taken out in certain time intervals to determine glucose concentration and calculate immobilized enzyme activity. Immobilization yield of ${ }^{\circ}$ Cactivity) was calculated as the ratio of activity of immobilized preparations and that of free glucoamylase used for immobilization.

Immobilized preparations ${ }^{\circ} \mathrm{C} 1 \mathrm{~mL}$ ) swelling sufficiently in $0.05 \mathrm{M}$ acetate buffer ${ }^{\circ} \mathrm{CpH} 4.5$ ) was first irradiated by UV light for $10 \mathrm{~min}$, and then was determined enzyme activity as above.

The storage stability of immobilized preparations was determined by measuring the activity of the preparations stored in $0.05 \mathrm{M}$ acetate buffer ${ }^{\circ} \mathrm{CpH}$ $4.5)$ at $4^{\circ} \mathrm{Cfor} 30$ days. The value of activity obtained directly after immobilization was set as $100 \%$.

\section{Results and Discussion}

\subsection{Carriers}

Five kinds of macroporous semi-interpenetrating stimuli-sensitive hydrogels, i.e. GelNIPAm-PAEMA, GelNIPAm-PHEMA, GelNDEP-PAEMA, GelNAEP-PAEMA and GelNAP-PAEMA, were prepared by incorporating a linear polymer of PAEMA or PHEMA into cross-linked polymer/copolymer of NIPAm, using PEG2000 as porogen. The specific figures of LCSTs and SRs of these hydrogels were showed in Table 2. It can be seen that all of them were thermo sensitive, which character should be help for the storage of immobilized enzyme and process control of using immobilized enzyme. The middle one is 
thermo and UV light dual-sensitive. The latter two are thermo, UV light and $\mathrm{pH}$ triply-sensitive. Both linear PAEMA and PHEMA are water-soluble so that the incorporation of them into gel network increased the hydrophilicity of the systems and the LCST and SR of those semi-interpenetrating hydrogels increased comparing with their conventional counterparts prepared without them [10]. For gelatinized starch, hydrophilic carrier would be help for the activity of immobilized glucoamylase [13]. So, it was believed that the incorporation of PAEMA or PHEMA into hydrogel systems would be more suitable for enzyme immobilization. Moreover, the incorporation of PAEMA will be help to the enzyme immobilization when using glutaraldehyde as cross-linking reagent because the primary amino groups in PAEMA could react with glutaraldehyde under mild conditions [14].

Table 2 the LCST and SR of semi-IPN hydrogels and their conventional counterparts

\begin{tabular}{lcccc}
\hline \multicolumn{1}{c}{ Hydrogels } & LCST $^{\mathrm{a}}$ & $\mathrm{SR}\left(30^{\circ} \mathrm{C}\right)$ & $\mathrm{SR}\left(30^{\circ} \mathrm{C}, \mathrm{UV}\right.$ irradiation $)$ & $\mathrm{SR}\left(45^{\circ} \mathrm{C}\right)$ \\
\hline GelNIPAm/PHEMA & 37.0 & 11.5 & - & 2.1 \\
GelNIPAm/PAEMA & 37.1 & 11.9 & - & 2.2 \\
GelNAEP/PAEMA & 35.4 & 7.69 & 8.54 & 1.95 \\
GelNAP/PAEMA & 37.2 & 8.73 & 9.18 & 2.17 \\
GelNDEP/PAEMA & 37.7 & 12.7 & 13.8 & 2.3 \\
\hline
\end{tabular}

aThe measure of LCST and SR referred to document [12].

\subsection{Immobilization of Glucoamylase}

The immobilization of glucoamylases was carried out in two steps. The first step is the adsorption of enzyme proteins on to hydrogel carriers. The absorption of a protein molecule on a solid surface is determined primarily by the surface properties. The hydrophobic surface of carriers is help to the adsorption of proteins. Therefore, the absorption of glucoamylase at the first stage proceeded at $45^{\circ} \mathrm{C}$, above carrier's LCST, when hydrophilic segments are buried within polymer network and hydrogels are hydrophobic. Next, the temperature was reduced quickly to the freezing point of water when carriers swelled and sucked glucoamylase rapidly. The second step is covalent reaction among enzyme protein or between enzyme protein and supporter using glutarldehyde as crosslinking agent. Carriers absorbed glucoamylase were treated with glutaraldehyde solution at $20^{\circ} \mathrm{C}$, three main reactions were carried out, i.e. reactions among carrier, enzyme molecule and glutaraldehyde, reactions between two enzyme molecules, and reactions between carrier and glutaraldehyde. So cross-linking with glutaraldehyde resulted in creation of a new interpenetrating network, i.e. the enzyme cross-linked with glutaraldehyde in carriers. The PAEMA interpenetrating networks was changed at the same time because enzyme would attach to the PAEMA network through 
glutaraldehyde. In addition, the glutaraldehyde molecule bound to the $\varepsilon$-amino groups of lysines of glucoamylases might covalently react with the glutaraldehyde molecule bound to the primary amino groups of the support to establish a multi-point covalent enzyme-support attachment in spite of the reaction was carried out in mild conditions.

\subsection{Activity of Immobilized Glucoamylase}

Table 3 Immobilization of glucoamylase on stimuli-hydrogel carriers ${ }^{\mathrm{a}}$

\begin{tabular}{|c|c|c|c|c|}
\hline Preparations & $\begin{array}{l}\text { Bound } \mathrm{p} \\
\mathrm{mg} / \mathrm{g}\end{array}$ & $\begin{array}{l}\text { Specific activity, } \\
\text { U/mg of protein }\end{array}$ & $\begin{array}{l}\text { Immobilization } \\
\text { yield }{ }^{\circ} \text { Cactivity), } \%\end{array}$ & $\begin{array}{l}\text { Storage } \\
\text { stability, \% }\end{array}$ \\
\hline GelNIPAm/PAEMA-0 & \multirow{4}{*}{5.61} & 7.7 & 5.70 & 23 \\
\hline GelNIPAm/PAEMA-1 & & 14.2 & 10.5 & 79 \\
\hline GelNIPAm/PAEMA-2.5 & & 15.4 & 11.4 & 81 \\
\hline GelNIPAm/PHEMA-2.5 & & 5.33 & 3.94 & 68 \\
\hline GelNIPAm -2.5 & 3.4 & 2.97 & 2.20 & 20 \\
\hline GelNAEP/PAEMA-2.5 & 5.62 & 17.2 & 12.7 & 80 \\
\hline GelNAP/PAEMA-2.5 & 5.58 & 20.1 & 14.9 & 84 \\
\hline GelNDEP/PAEMA-2.5 & 5.87 & 20.7 & 15.3 & 87 \\
\hline
\end{tabular}

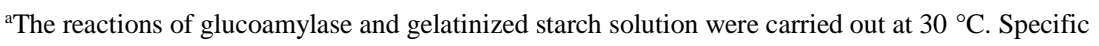
activity of free glucoamylase in the presence of starch: $135.2 \mathrm{U} / \mathrm{mg}$

Properties of the obtained preparations are shown in Table 3. The number in every preparation means the concentration of glutaraldehyde solution used for immobilized glucoamylase. It can be seen that almost the same quantity of protein was loaded for GelNIPAm-PAEMA carriers, but the activities of those immobilized glucoamylases increased with increasing of cross-linker. For GelNIPAm-PAEMA-0, there were no glutaraldehyde attendance; most of the adsorbed enzymes were attached to hydrophobic groups so that many of them were buried within polymer network at temperature below their LCST which hindered the starch molecular reach to enzymes. On the contrary, when enzymes were immobilized on the amino-groups of PAEMA with glutaraldehyde, they became more available because of the hydrophilic of the amino-groups. So the more cross-linker used the more accessible enzymes were obtained, and the highest value of activity, $15.4 \mathrm{U} / \mathrm{mg}$, was obtained for carrier cross-linked with $2.5 \mathrm{vol} \%$ of glutaraldehyde. GelNIPAm-PAEMA-2.5's immobilization yield ${ }^{\circ}$ Cactivity) was $11.4 \%$ higher than some reported results $[6,11]$ which were possibly attribute to the macroporous semi-interpenetrating network structure of hydrogel carrier. The activity of GelNIPAm-PAEMA-2.5 was better than that of GelNIPAm-PHEMA-2.5 because there were no amino-groups in PHEMA so that the cross-linking were happen among enzyme molecules which resulted in a short distance between two enzyme molecules, consequently starch 
molecular have been limited to access the active site. In addition, the least quantity of glucoamylase protein was loaded for GelNIPAm-2.5 and the worst activity was obtained, the possible reason was that the hydrophilic of GelNIPAm-2.5 was te worst among all of the composited semi-interpenetrating hydrogels ${ }^{\circ} \mathrm{CTable} 1$ ) which leaded to few enzyme molecules adsorbed. Second, the cross-linking was happen among enzyme molecules which resulted its' unaccessible. For preparations of GelNAEP-PAEMA-2.5, GelNAP-PAEMA-2.5 and GelNDEP-PAEMA-2.5, the bound enzyme protein was similar to that of GelNIPAm-PAEMA carriers, and good activities were obtained,i.e. 17.2 U/mg, $20.1 \mathrm{U} / \mathrm{mg}$ and $20.7 \mathrm{U} / \mathrm{mg}$ respectively, which might attribute to the join of stimulus response comonomers AEPABA, APABA and DMAE-EAPP, they would change the pore structure of PNIPAm [10].

\subsection{Activity of Immobilized Glucoamylase in Different Reaction Condition}

Here, the affection of temperature, UV light irradiation and $\mathrm{pH}$ of reaction system on the activities of immobilized glucoamylases were studied carefully. The results were showed in Fig. 1. With increasing temperature, the activity of free glucoamylse increased first and then decreased. The best value of activity was $145.8 \mathrm{U} / \mathrm{mg}$ at $40{ }^{\circ} \mathrm{C}$. When glucoamylases were immobilized on different hydrogel carriers their activities reduced greatly. There are two possible reasons: First, enzyme proteins should undergo conformational changes during adsorption. When the enzyme was immobilized on the primary amino groups of the support by covalent bond, some even were muti-point attached, which stiffened the enzyme conformation and resulted in distortion of the enzyme molecules, so the immobilized enzyme inevitably should lost some of their biological activity [15]. Second, the activity of immobilized enzyme was reduced by mass transfer effects because of the block of the polymer network. In spite of this, good immobilized yields ${ }^{\circ}$ Cactivity) were obtained. The adapted reaction temperature was shift to $\sim 30^{\circ} \mathrm{C}$ which should attribute to the thermo sensitive character of carrier. The LCST of all the carriers were between $35.4{ }^{\circ} \mathrm{C}$ and $37.7{ }^{\circ} \mathrm{C}$ which imply the shrink of hydrogels begin at some temperature higher than $30{ }^{\circ} \mathrm{C}$ when the accesses of starch substrate to the active sites become difficult. Comparing the values of activity of preparations, GelNIPAm/PAEMA-2.5, GelNAEP/AEMA-2.5 and GelNAP/PAEMA-2.5, the order was GelNDEP/PAEMA-2.5> GelNAP/PAEMA-2.5 > GelNAEP/PAEMA-2.5, which was just accordance with the order of their LCST from high to low. In addition, the values of activity of GelNIPAm/PAEMA-2.5 were lower than that of GelNAEP/PAEMA-2.5 at same temperature though the 
hydrophilic of former was better than the latter, which may be attributed to the change of porous structure resulted by their comonomers.

The activities of GelNAEP/PAEMA-2.5, GelNAP/PAEMA-2.5 and GelNDEP/PAEMA-2.5 increased to some extent after UV irradiation during their phase transition. Upon UV irradiation, the hydrophobic trans-azobenzene chromophores can switch to relatively hydrophilic cis configuration which should improve the hydrophility of their copolymers so that the carrier was swelling and then the starch substrate could attach the immobilized enzyme. The result showed that the activity of these immobilized glucoamylases on photo-sensitive hydrogel carriers could be adjusted by UV irradiation.
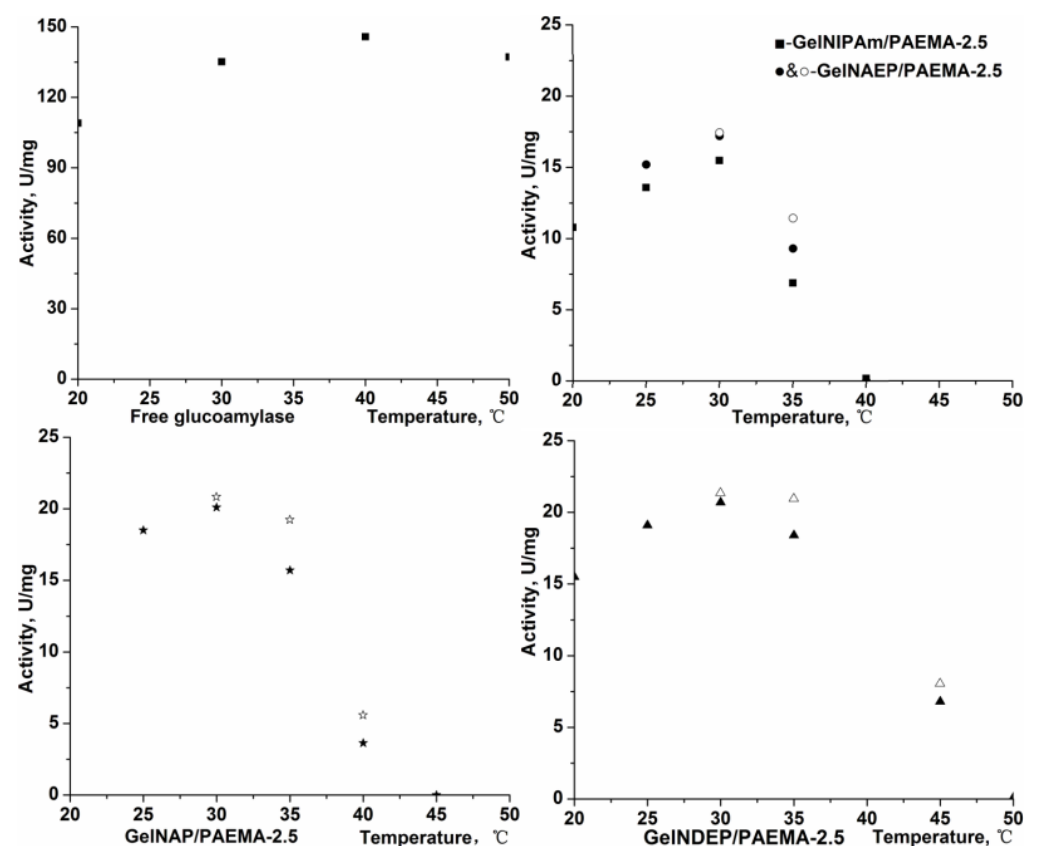

Fig. 1 Specific activities of free and immobilized glucoamylase at different temperature. Bold dotenzyme activity test without UV irradiation, and hollow dot- enzyme activity after UV irradiation

Different $\mathrm{pH}$ condition were studied for those immobilized enzymes, the results showed that the activity hardly increased with decreasing the $\mathrm{pH}$ of reaction system, but it should reduce distinctly when increasing the value of $\mathrm{pH}$ of reaction mixture. The optimum $\mathrm{pH}$ of immobilized ones was $\mathrm{pH} 4.5$. The affection of reaction system $\mathrm{pH}$ would be studied carefully in our later work. 


\subsection{Storage Stability of Immobilized Glucoamylase}

The storage stability was showed in table 3 row 5. For Gel NIPAm/PAEMA series preparations, the storage stability increases with the increase of glutaraldehyde concentration used. The possible reason was: for GelNIPAm/PAEMA-0, the enzyme molecules were physically absorbed on the surface of the solids so that they were easy to run off during storage. When some glutaraldehydes were used the glucoamylases were bounded by covalent on PAEMA. The activity was kept $68 \%$ for GelNIPAm/PHEMA- 2.5 which was less than that of GelNIPAm/PAEMA-2.5 which may attribute to more multi-point covalent cross-linking among enzyme molecules and some escape during storage. Worst storage stability was checked for GelNIPAm-2.5 which may attributed to multi-point covalent cross-linkingand serious escape during storage because the macroporous poly ${ }^{\circ} \mathrm{CN}$-isopropylacrylamide) network were free enough to flee without PHEMA as semi-interpenetrating network. Good activity retentions of GelNAEP/PAEMA-2.5, GelNAP/PAEMA-2.5 and GelNDEP/PAEMA-2.5 were $80 \%, 84 \%$ and $87 \%$ respectively.

\section{Conclusions}

Five kinds of macroporous semi-interpenetrating stimuli-sensitive hydrogels were synthesized, i.e. GelNIPAm-PAEMA, GelNIPAm-PHEMA, GelNDEPPAEMA, GelNAEP-PAEMA and GelNAP-PAEMA. Taking them as carries, five immobilized glucoamylase preparations were prepareds. The study showed that almost the same quantity of protein was loaded for the five macroporous semi-interpenetrating hydrogel carriers, but their activities were different. The activity increased with increasing concentration of cross-linker used and was also related to the hydrophility, stimuli-sensitivity, and microstructure of carriers. The most preferred temperature of immobilized ones was $30{ }^{\circ} \mathrm{C}$, whilst the adapted $\mathrm{pH}$ was $\mathrm{pH} 4.5$. Four good preparations were screened, i.e. GelNIPAm/PAEMA-2.5, GelNAEP/PAEMA-2.5, GelNAP/ PAEMA-2.5 and GelNDEP/PAEMA-2.5, the immobilization yield ${ }^{\circ}$ Cactivity) of them were $11.4 \%, 12.7 \%, 14.9 \%$ and $15.3 \%$ respectively under optimal conditions which were better than some reported results. In addition, the activities of GelNAEP/PAEMA-2.5, GelNAP/PAEMA-2.5 and GelNDEP/PAEMA-2.5 could be adjusted by UV irradiation during their phase transition. The most stable immobilized glucoamylase are preparations cross-linked with 2.5 vol\% of glutaraldehyde. Activity retentions of four good preparations were above $81 \%$. 


\section{References}

1. R.R. Singhania, A.K. Patel, R.K. Sukumaran, C. Larroche and A. Pandey, Role and significance of beta-glucosidases in the hydrolysis of cellulose for bioethanol production, Bioresource Technol. 127: 500-507 ${ }^{\circ} \mathrm{C} 2013$ ).

2. T. G. Argun, and A. Esra, Synthesis and investigation of poly ${ }^{\circ}$ CNIPAMco-N-vinylcarbazole) hydrogels morphological, fluorescence and electrical properties, Journal of Molecular Structure, 1108: 25-32 $\left.{ }^{\circ} \mathrm{C} 2016\right)$.

3. L. Sumitra Datta, R. Christena and R. Yamuna, Enzyme immobilization: an overview on techniques and support materials, Biotech 3:1-9 ${ }^{\circ} \mathrm{C} 2013$ ).

4. R. Debashish, J. N. Cambre and B. S. Sumerlin, Future perspectives and recent advances in stimuli-responsive materials. Progress in Polymer Science, 35: 278-301 $\left.{ }^{\circ} \mathrm{C} 2010\right)$.

5. M.O. Zakir, S. D. Rzaev and P. Erhan, Functional copolymers of N -isopropylacrylamide for bioengineering applications [J]. Prog. Polym. Sci. 32: 534-595 $\left.{ }^{\circ} \mathrm{C} 2007\right)$.

6. H.-D. Agnieszka, J. Bryjak and A. W. Trochimczuk. Immobilization of glucoamylase and trypsin on crosslinked thermosensitive carriers [J]. Enzyme and Microbial Technology, 2007, 41: 197-204.

7. M. Nikola, M. Nedeljko, F. Jovanka and K. Zorica, Synthesis, characterization and application of poly ${ }^{\circ} \mathrm{CN}$ -isopropylacrylamide-co-itaconic acid) hydrogels as supports for lipase immobilization. Reactive \& Functional Polymers 70: 807-814 ${ }^{\circ} \mathrm{C} 2010$ ).

8. Y. Liang, Sh. Song, H. Yao and N. Hu, Triply switchable bioelectrocatalysis based on poly ${ }^{\circ} \mathrm{C}$-isopropylacrylamide) hydrogel films with immobilized glucose oxidase, Electrochimica Acta 56: 5166-5173 ${ }^{\circ} \mathrm{C} 2011$ ).

9. G. Cirillo, F. P.e Nicoletta, M. Curcio, U. G. Spizzirri, N. Picci and F. Iemma, Enzyme immobilization on smart polymers: Catalysis on demand. Reactive \& Functional Polymers, 83: 62-69 $\left.{ }^{\circ} \mathrm{C} 2014\right)$.

10. L. Li, B. Lu, Y. Zhang, X. Xing, X. Wu and Z. Liu. Multi-sensitive copolymer hydrogels of $\mathrm{N}$-isopropylacrylamide with several polymerizable azobenzene-containing monomers. J Polym Res, 22: 176-188 $\left.{ }^{\circ} \mathrm{C} 2015\right)$.

11. H.-D. Agnieszka, J. Bryjak and A. W. Trochimczuk, Novel method of enzymes stabilization on crosslinked thermosensitive carriers, Enzyme and Microbial Technology 38: 921-925 $\left.{ }^{\circ} \mathrm{C} 2006\right)$.

12. L. Li, X. Xing and Z. Liu. Triply-responsive ${ }^{\circ}$ Cthermo/light/pH) copolymeric hydrogel of $\mathrm{N}$-isopropylacrylamide with an azobenzene-containing monomer, Journal of Applied Polymer Science, 124: $\left.1128-1136{ }^{\circ} \mathrm{C} 2012\right)$.

13. R. A. Messing. Carriers for immobilized biologically active systems, Advances in Biochemical Engineering, 10: 51-73 $\left.{ }^{\circ} \mathrm{C} 2005\right)$. 
14. L 'opez-Galego F, Montes T, Fuentes M, Alonso $\mathrm{N}$ and Betancor L. Improved stabilization of chemically aminated enzymes via multipoint covalent attachment on glyoxyl supports, J Biotechnol,116: 1-10 $\left.{ }^{\circ} \mathrm{C} 2005\right)$.

15. A. Garland, L. Shen, X. Zhu, Mobile precursor mediated protein adsorption on solid surfaces, Progress in Surface Science, 87: 1-22 ${ }^{\circ}$ C2012). 